\title{
Prospective cohort study using the breast cancer spheroid model as a predictor for response to neoadjuvant therapy - the SpheroNEO study
}

Kathrin Halfter ${ }^{1 \dagger}$, Nina Ditsch ${ }^{2 \dagger}$, Hans-Christian Kolberg ${ }^{3}$, Holger Fischer $^{4}$, Tanja Hauzenberger ${ }^{5}$, Franz Edler von Koch ${ }^{6}$, Ingo Bauerfeind ${ }^{7}$, Gunter von Minckwitz ${ }^{8}$, llona Funke ${ }^{9}$, Alexander Crispin ${ }^{10}$, Barbara Mayer ${ }^{1,9^{*}}$ and Behalf of the SpheroNEO Study Group

\begin{abstract}
Background: Aim of this prospective study was to predict response to neoadjuvant therapy in breast cancer patients using an in vitro breast cancer spheroid model.

Methods: Three-dimensional spheroids were directly generated from fresh breast tumor biopsies of 78 patients eligible for neoadjuvant therapy. Cell survival was measured after in vitro exposure to the equivalent therapeutic agents in the breast cancer spheroid model. Treatment results in vitro were correlated with pathological complete response ( $p C R$, i.e. ypTO ypNO) determined at surgery.
\end{abstract}

Results: A mean cell survival of $21.8 \%$ was found in the breast cancer spheroid model for 22 patients with pCR versus $63.8 \%$ in 56 patients without $p C R(P=.001)$. The area under the receiver operator characteristic curve to predict PCR was 0.86 (95\% Cl: 0.77 to 0.96 ) for cell survival in vitro compared to 0.80 ( $95 \% \mathrm{Cl}: 0.70$ to 0.90 ) for a combined model of conventional factors (hormone- and HER2 receptor, and age). A cutoff at $35 \%$ cell survival for the spheroid model was proposed. Out of the 32 patients with values below this threshold, 21 patients (65.6\%) and one patient (2.2\%) with a cell survival greater than $35 \%$ achieved pCR respectively; (sensitivity $95.5 \%$ (95\% Cl: 0.86 to 1.00$)$; specificity $80.4 \%$ (95 \% Cl: 0.70 to 0.91$)$ ). Extent of residual disease positively correlated with increased cell survival $(P=.021)$.

Conclusion: The breast cancer spheroid model proved to be a highly sensitive and specific predictor for pCR after neoadjuvant chemotherapy in breast cancer patients.

Keywords: Breast cancer, Neoadjuvant chemotherapy, Predicting treatment outcome, Cellular response to anticancer drugs, Personalized medicine, in vitro diagnostics

\section{Background}

Multiple studies in breast cancer have shown that pathological complete response $(\mathrm{pCR})$ serves as a reliable surrogate marker for progression-free survival, as well as overall survival [1-6]. Despite all efforts, the rate of pathological complete response (pCR) following neoadjuvant therapy for primary breast cancer remains low at an average rate of $20-30 \%[1,7]$. Efforts to maximize

\footnotetext{
* Correspondence: Barbara.mayer@med.uni-muenchen.de

${ }^{\dagger}$ Equal contributors

'Department of General, Visceral, Transplantation, Vascular and Thoraic Surgery, Hospital of the University of Munich, Munich, Germany

${ }^{9}$ SpheroTec GmbH, Martinsried, Germany

Full list of author information is available at the end of the article
}

the outcome of the standard neoadjuvant treatment have been tested in numerous clinical trials, with variations in dosing such as dose-dense or dose-intensified regimen [8], or order of application of single-agent and combination treatment regimen $[9,10]$. In addition, established cytostatic agents or new drugs targeting HER2, angiogenesis, or mammalian target of Rapamycin (mTOR) have been combined to novel therapy strategies [11-16]. The application of targeted therapy in combination with a taxane/anthracycine-based regimen with the addition of carboplatin resulted in an increase of the rate of $\mathrm{pCR}$ by $16.3 \%$ in triple negative breast cancer patients [17]. Other trials have not yielded such promising results or 
the data obtained was inconclusive and did not warrant changes in guideline recommendations. The addition of capecitabine to a standard anthracycline/taxane-based regimen [14], the sequence of paclitaxel and 5-FU/ anthracycline/cyclophosphamide combination therapy [10], or the treatment of early non-responders with the mTOR-inhibitor everolimus given simultaneously with paclitaxel [15] did not show any improvement of pCR. Encouraging developments have been made regarding single and dual agent anti-HER2 blockade. This treatment option raised the rate of $\mathrm{pCR}$ among women with HER2-positive tumors up to $66.7 \%$ [18-20]. Unfortunately, among the HER2 positive patient population only a limited proportion show a treatment response to HER2 inhibition [21, 22]. In addition, only $20 \%$ of all tumors diagnosed are positive for this targetable biomarker [23], leaving the majority of women with no further treatment options aside from the standard chemotherapy treatment. So far, no biomarker has been accepted for routine use to accurately predict treatment outcome to chemo- and/or anti-HER2-therapy.

Currently predictive diagnostic tests are available that stratify patients to an individual treatment regimen using a genetic or cellular approach. Assays based on the genetic analysis of the tumor tissue make up the largest proportion; some of these tests have already been validated for specified subgroups in prospective trials [24-26]. However, no data that would indicate the most effective treatment option is provided even though numerous treatment options in breast cancer treatment are available [24, 27]. Cell-based chemosensitivity and chemoresistance assays, such as single-cell suspension and cell monolayer assays [28-31], are currently not sufficiently validated for clinical implementation [32]. Contrary to the 2-Dimensional (2D) cell culture models, 3-Dimensional (3D) spheroid models reflect the tumor biology and tumor microenvironment much more accurately [33-38]. The spheroid-based assay proposed herein, was assessed as a diagnostic tool to aid in the therapeutic decision-making. The objective of the SpheroNEO study was to test whether in vitro treatment results obtained in the breast cancer spheroid model are associated with treatment outcome in primary breast cancer patients undergoing neoadjuvant therapy.

\section{Methods}

Starting from October 2009 until September 2012, 202 patients from 13 breast cancer centers in Germany were enrolled in the SpheroNEO study. Written consent for the trial was given by all applicable ethics committees. An informed consent was obtained from all eligible patients (Additional file 1). Patients 18 years or older were eligible if a clinically confirmed case of invasive breast cancer had been diagnosed and the use of neoadjuvant chemotherapy was recommended. Patients with a previous diagnosis and/or treatment of a malignant disease, as well as patients with metastatic disease were excluded.

\section{Study design}

The SpheroNEO study was designed as a prospective, non-interventional cohort study. Tumor tissue from core needle biopsies was obtained simultaneously for the SpheroNEO study and histopathological diagnosis. Drugs tested in the breast cancer spheroid model were recommended by the treating physicians of the breast cancer center at the time of the biopsy procedure. Results obtained in the breast cancer spheroid model had no impact on the treatment decision for the individual patient, and treating physicians were blinded to the results of the laboratory test. A comparison of the therapeutic response in vitro and clinical treatment outcome documented in the pathological report was performed after all patients had completed the neoadjuvant treatment followed by surgery. Pathological complete response was defined as no vital tumor in breast or axilla (pCR, i.e. ypT0 ypN0) determined at surgery following the completion of chemotherapy.

\section{Breast cancer spheroid model}

Fresh tumor biopsy samples were collected in freshly prepared culture medium containing DMEM/F12-medium (PAN), $10 \%$ fetal calf serum (PAN), 2x MEM nonessential amino acid solution (PAN), 2x MEM vitamin solution NEAA, as well as a mixture of antibiotic/antifungal compounds $(0.26 \mu \mathrm{M}$ Amphotericin B, Ampicillin $0.14 \mathrm{mM}$, Ciprofloxacin $7.54 \mu \mathrm{M}$ ) and shipped from participating breast cancer centers to an external laboratory. All laboratory procedures and tests were performed according to standardized, quality-controlled handling procedures. The tumor samples underwent mechanical and enzymatic digestion using an enzyme cocktail containing Liberase TM, which consisted of a mixture of collagenases and neutral protease enzymes (Roche, Penzberg Germany). After determination of cell viability using the trypan-blue exclusion test, the single cell suspension was directly processed into breast cancer spheroids using a modified liquid overlay technique $[39,40]$. No red blood cell lysis was performed. A training cohort of 14 breast tumor biopsies was tested prior to study start, to optimize the assay protocol. This was necessary since the previously described liquid overlay method used RPMI culture medium for basic cell line culture, this was replaced by DMEM/F12 to better accommodate primary tumor cells. The cell isolation procedure was also supplemented to include additional washing steps to filter out cellular debris. For this purpose the use of a cell strainer was added to the cell isolation procedure as well. 
The spheroids were cultured for $48 \mathrm{~h}$ at $37{ }^{\circ} \mathrm{C}$ and $5 \%$ $\mathrm{CO}_{2}$ and treated with the recommended combination of cytostatic agents using the peak plasma concentration of each drug (ppc), see Table 1 for the utilized concentrations and solvent controls. Spheroid formation was verified and grade of compaction was documented using bright field microscopy prior to drug treatment. Medium was not changed during any time during the spheroid culture. The duration of the drug treatment was $96 \mathrm{~h}$ after which the treatment efficacy was assessed using a standard assay measuring metabolic activity (Promega, Mannheim Germany) to quantify cell survival in vitro. Mean cell survival was expressed as percent residual metabolic activity of the respective solvent controls. Laboratory test results were available after eight days.

\section{Statistics}

Sample size was estimated based on a $95 \%$ true-positive rate (TPR) and a true negative rate (TNR) of $90 \%$ for the breast cancer spheroid model. If $20 \%$ of the patients achieved pCR, a sample size of 70 was regarded as sufficient to estimate both TPR and TNR with a lower limit of the $95 \%$ Clopper-Pearson interval greater than $66 \%$.

All results are reported from the intention-to-treat analysis. Clinical and laboratory data was described separately for patients with and without pCR using appropriate measures of location and dispersion. Analyses of the discriminatory power of the spheroid model and traditional risk factors were based on receiver operating characteristic (ROC) curves and the corresponding c statistics (areas under the ROC curves, AUC) calculated using the SAS LOGISTIC procedure. Additional logistic models were fit to estimate the role of the assay results in the context of traditional risk factors, using forward selection based on Wald tests on an alpha level of $5 \%$. Since this frequently lead to a disproportion between the number of the covariates and the sample size, the

Table 1 Cytostatic compounds used in the breast cancer spheroid model

\begin{tabular}{lrl}
\hline Name & $\begin{array}{l}\text { PPC conzentration } \\
{[\mu \mathrm{g} / \mathrm{ml}]}\end{array}$ & Literature source \\
\hline Carboplatin & 40.843 & Go, Adjei, 1999 [53] \\
Cyclophosphamid & 41.000 & Egorin et al, 1989 [54] \\
Docetaxel & 2.180 & Baker et al, 2004 [55]; \\
& & Bruno et al, 1998 [56] \\
Doxorubicin & 1.640 & Brana et al, 2014 [57] \\
Epirubicin & 1.005 & Reviewed in Fujimoto, 2007 [58] \\
Fluorouracil (5-FU) & 100.000 & Reviewed in Fujimoto, 2007 [58] \\
Paclitaxel & 1.530 & Gianni et al, 1995 [59] \\
Trastuzumab & 88.000 & Leyland-Jones, 2001 [60] \\
\hline
\end{tabular}

analysis was replicated using penalized (lasso) logistic regression in the R package "penalized". DeLong $95 \%$ confidence intervals for the $\mathrm{c}$ statistics from the lasso regression models were calculated using the $\mathrm{R}$ package 'pROC'. The 95th percentile of the residual activity in women with pCR was used as cut-off and the resulting sensitivity and specificity and their $95 \%$ confidence limits are reported, calculation of the Youden Index resulted in the same value. Laboratory data was correlated with pCR using Pearson's chi-square or Fisher's exact tests for categorical factors and t-tests for numerical variables. The correlation between cell survival in vitro after treatment and the size of residual tumor in the breast (ypT) after neoadjuvant therapy was quantified using Spearman's rank correlation coefficient. All hypotheses tested were two-sided on an alpha level of $5 \%$. Analyses were performed using the Statistical Analysis System SAS, version 9.2 for Linux (SAS Institute, Cary, NC), as well as $\mathrm{R}$ version 2.12.2 for Windows (The R Foundation for Statistical Computing).

\section{Results}

A total of 202 patients were enrolled in the SpheroNEO study. Figure 1 shows in detail the exclusion criteria for the screened patients. The main clinical reasons for exclusion were both related to choice of treatment. This was due to the fact that at the time of biopsy the results from the staging examinations were not yet available, and a definitive treatment decision had not been made. A total of 30 patients did not receive neoadjuvant treatment as initially planned, instead undergoing primary surgery followed by adjuvant treatment. The second main reason for exclusion $(n=21)$ was the discrepancy between clinical therapy and in vitro treatment, due to results from final staging examinations or patient preferences.

As to the laboratory criteria, the main reason for exclusion was the limited amount of cells isolated from the biopsies. Due to the limited number of available cells no additional assays were possible. This resulted in an insufficient number of isolated cells (calculated minimum of 2841 cells required; $n=13$ ) or a metabolic activity below threshold $(n=32)$. The mean duration from biopsy procedure to the start of tissue preparation was $26.4 \mathrm{~h}$ (range 1.0 - $162.8 \mathrm{~h}$ ). A breast cancer spheroid assay was considered successful when the minimal metabolic of the untreated controls measured at least 107.5 counts per second (cps). This threshold represented twice the mean luminescence for the solvent controls without cells. Results show that a minimum of four biopsy cylinders with a mean total weight of $89.6 \mathrm{mg}$ (range 10.5 - 353.4 $\mathrm{mg}$ ), were required to test a minimum of three treatment combinations. The final analysis included 78 patients, who qualified according to both clinic and laboratory criteria. 


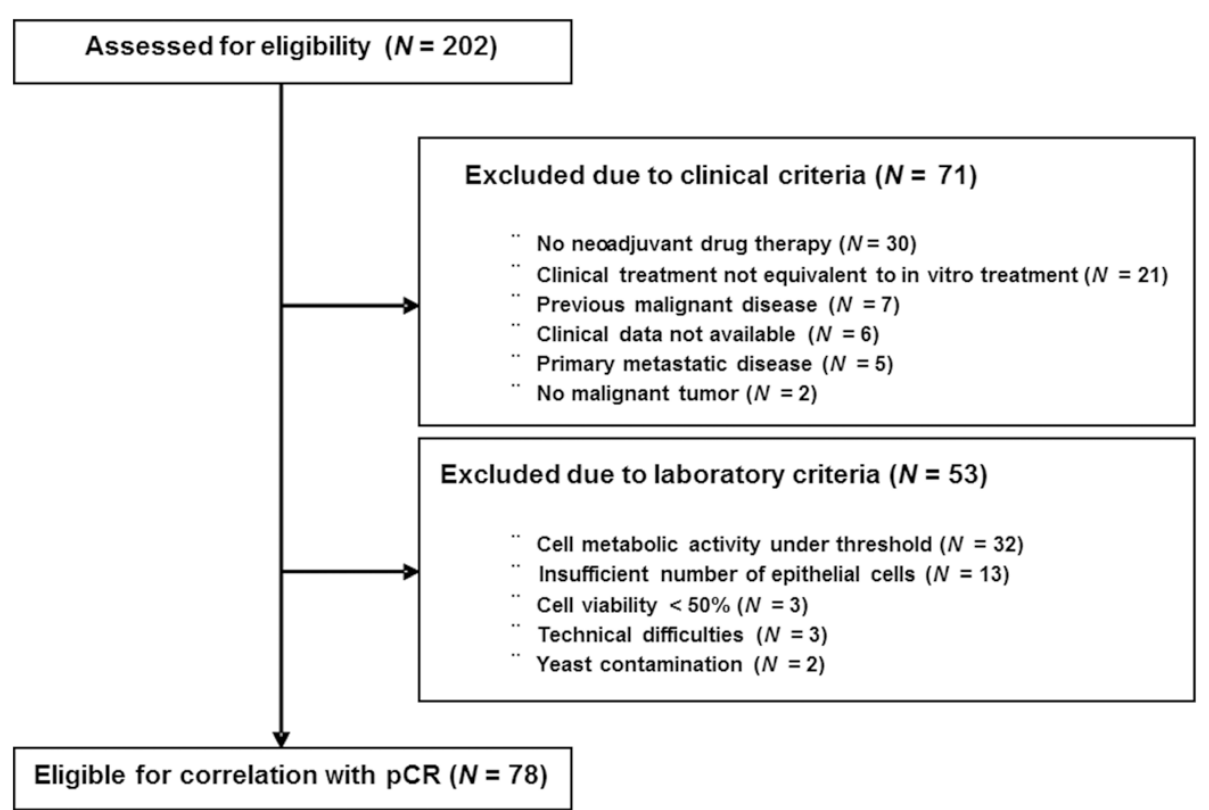

Fig. 1 Study flowchart depicting the screening process of the patients in the SpheroNEO cohort

Baseline characteristics of the SpheroNEO study cohort Baseline characteristics are shown in Table 2. Age, clinical tumor stage, tumor histology, and receptor status were representative compared to previously published larger cohorts $[6,41]$. All except two patients received taxane-based chemotherapy combined with either anthracycline or carboplatin. All patients with HER2-positive tumors received trastuzumab-based therapy (20.5\%). Patient tumors were most frequently invasive ductal in their histology (61 out of $78 ; 78.2 \%$ ) with a median diameter of $2-5 \mathrm{~cm}$ (cT2; 45 out of 77; $58.4 \%)$. Regarding tumor biology, $64.5 \%$ (49 out of 76) and $53.2 \%$ (42 out of 77) were positive for estrogen- and progesterone receptor respectively.

The overall pCR rate for the SpheroNEO study cohort was $28.2 \%$ (22 out of 78). This rate is similar to other studies where the majority of patients were also treated with an anthracycline/taxane-based regimen [3, 42]. As expected, pCR rate was higher in younger patients, hormone receptor negative patients, as well as HER2positive patients (Table 2). Treatment non-adherence defined as treatment discontinuation, dose-reduction, or change of treatment regime resulted in a lower $\mathrm{pCR}$ rate $(p=.016)$.

\section{Characterization of the breast cancer spheroid model}

Tumor spheroids were directly derived from the tissue samples without selecting any specific cell type [43]. Due to the heterogeneity of the tissue, the breast cancer spheroids varied between each patient in regard to cellular composition. The average size and compaction of the spheroids was dependent on the number of cells per spheroid and the cellular composition. Spheroid morphology was classified as compact, intermediate, and loose as previously published [40] and correlated with the patient characteristics. The spheroid morphology was not effected by age $(p=.678)$, cT Stadium $(p=.064)$, nodal status $(p=.473)$, hormone receptor $(p=.256)$ or HER2 status $(p=.082)$, as well as Ki67 ( $\mathrm{p}=.536)$. A lobular histology also did not have an impact on spheroid morphology, however only 8 cases were included in the main analysis. The only factor which was associated with significant differences in spheroid morphology was the grading of the tumor $(p=.009)$. High grade tumors tended to form less compact spheroids while low grade tumors generated more compact spheroids.

\section{Predictive power of the breast cancer spheroid model for treatment outcome}

A significant difference in cell survival in vitro was found in a comparison of patients achieving pCR versus those who did not ( $p=.001$; Fig. 2). A mean cell survival of $21.8 \%$ was found in the breast cancer spheroid model for patients with pCR versus $63.8 \%$ in non-pCR patients. For trastuzumab-based regimen a mean of $21.9 \%$ versus $45.4 \%$ was seen in patients achieving or not achieving pCR respectively $(p=.085)$.

Hormone-receptor negative tumor samples (43.03\%, $p=.038$ ) and HER2-positive tumor samples (30.7\%, $p<.0001)$ showed lower cell survival rates than hormonereceptor-positive or HER2-negative tumor samples, respectively (Table 3 ). 
Table 2 Baseline Characteristics and pCR Rates of the SpheroNEO cohort

\begin{tabular}{|c|c|c|c|c|c|c|}
\hline \multirow[b]{3}{*}{ Characteristics } & & & \multicolumn{4}{|c|}{$\mathrm{pCR}$} \\
\hline & \multicolumn{2}{|c|}{ All Patients } & \multicolumn{2}{|c|}{ Yes } & \multicolumn{2}{|r|}{ No } \\
\hline & $n$ & $\%$ & $\bar{n}$ & $\%$ & $\bar{n}$ & $\%$ \\
\hline All Patients & 78 & 100 & 22 & 28.2 & 56 & 71.8 \\
\hline \multicolumn{7}{|l|}{ Age at diagnosis, years } \\
\hline$\leq 50$ & 43 & 55.1 & 15 & 34.9 & 28 & 65.1 \\
\hline$>50$ & 35 & 44.9 & 7 & 20.0 & 28 & 80.0 \\
\hline Mean & \multicolumn{2}{|c|}{$5121-78$} & \multicolumn{2}{|c|}{$4621-65$} & \multicolumn{2}{|c|}{$5325-78$} \\
\hline \multicolumn{7}{|l|}{ Range } \\
\hline \multicolumn{7}{|l|}{ Tumor stage } \\
\hline $\mathrm{CT} 1 / \mathrm{T} 2$ & 56 & 72.7 & 16 & 28.6 & 40 & 71.4 \\
\hline $\mathrm{CT} 3 / 4$ & 21 & 27.3 & 5 & 23.8 & 16 & 76.2 \\
\hline Not documented & 1 & - & & & & \\
\hline \multicolumn{7}{|l|}{ Nodal status } \\
\hline $\mathrm{cN}+$ & 42 & 54.5 & 12 & 28.6 & 30 & 71.4 \\
\hline $\mathrm{cN}-$ & 35 & 45.5 & 10 & 28.6 & 25 & 71.4 \\
\hline Not documented & 1 & - & & & & \\
\hline \multicolumn{7}{|l|}{ Grading } \\
\hline $\mathrm{G} 1 / 2$ & 41 & 53.9 & 8 & 19.5 & 33 & 80.5 \\
\hline G3 & 35 & 46.1 & 13 & 37.1 & 22 & 62.9 \\
\hline Not documented & 2 & - & & & & \\
\hline \multicolumn{7}{|l|}{ Histologic type } \\
\hline Invasive ductal/other & 70 & 89.7 & 21 & 30.0 & 49 & 70.0 \\
\hline Invasive lobular & 8 & 10.0 & 1 & 12.5 & 7 & 87.5 \\
\hline \multicolumn{7}{|l|}{ HR status } \\
\hline $\mathrm{ER}+/ \mathrm{PR}+$ & 39 & 52.0 & 4 & 10.3 & 35 & 89.7 \\
\hline ER+/PR-/Unknown & 10 & 13.3 & 7 & 70.0 & 3 & 30.0 \\
\hline ER-/unknown/PR+ & 2 & 2.7 & 1 & 50.0 & 1 & 50.0 \\
\hline ER-/PR- & 24 & 32.0 & 9 & 37.5 & 15 & 62.5 \\
\hline Not documented & 3 & - & & & & \\
\hline \multicolumn{7}{|l|}{ HER2 status } \\
\hline Negative & 59 & 78.7 & 11 & 18.6 & 48 & 81.4 \\
\hline Positive & 16 & 21.3 & 10 & 62.5 & 6 & 37.5 \\
\hline Not documented & 3 & - & & & & \\
\hline \multicolumn{7}{|l|}{ Drug Therapy } \\
\hline $\mathrm{AC} \rightarrow \mathrm{T}$ & 57 & 71.8 & 11 & 19.3 & 46 & 80.7 \\
\hline $\mathrm{AC} \rightarrow \mathrm{TH}$ & 9 & 11.5 & 8 & 88.9 & 1 & 11.1 \\
\hline $\mathrm{TCbH}$ & 7 & 9.0 & 2 & 28.6 & 5 & 71.4 \\
\hline $\mathrm{AC} \rightarrow \mathrm{TCb}$ & 3 & 3.8 & 1 & 33.3 & 2 & 66.7 \\
\hline$A C$ & 2 & 3.6 & 0 & 0.0 & 2 & 100.0 \\
\hline
\end{tabular}

Table 2 Baseline Characteristics and pCR Rates of the SpheroNEO cohort (Continued)

\begin{tabular}{llllllll}
\hline $\begin{array}{l}\text { Treatment adherence } \\
\text { Yes }\end{array}$ & 60 & 76.9 & 21 & 35.0 & 39 & 65.0 & \\
No & 18 & 23.1 & 1 & 5.6 & 17 & 94.4 & \\
\hline
\end{tabular}

Statistical tests for categorical factors were performed using the Pearson's $\mathrm{X}^{2}$ test or Fisher's exact test; tests for numerical factors were performed using a t-test

$\mathrm{PR}$, progesterone receptor; $\mathrm{ER}$, estrogen receptor; HER2, human epidermal growth factor receptor; $\mathrm{PCR}$, pathologic complete response; $\mathrm{A}$, anthracycline, $\mathrm{T}$, paclitaxel or docetaxel, $\mathrm{C}$, cyclophosphamide; $\mathrm{Cb}$, carboplatin, $\mathrm{H}$, trastuzumab

The predictive power of the breast cancer spheroid model was analyzed in the context of established predictors. The c statistic (area under the ROC curve) for the treatment results in the breast cancer spheroid model was 0.86 (Fig. 3a), which was superior to classic risk factors. The AUC for the standard clinical factors significantly correlated with $\mathrm{pCR}$ in this cohort which were hormone-receptor status, HER2, and age resulted in an AUC of 0.80 (Fig. 3b). Simultaneous modelling of the effects of the spheroid model and clinical predictors in lasso regression models resulted in a slight increase of the AUC to 0.91 when HER2 and the type of therapy were considered, since patients receiving a trastuzumab-

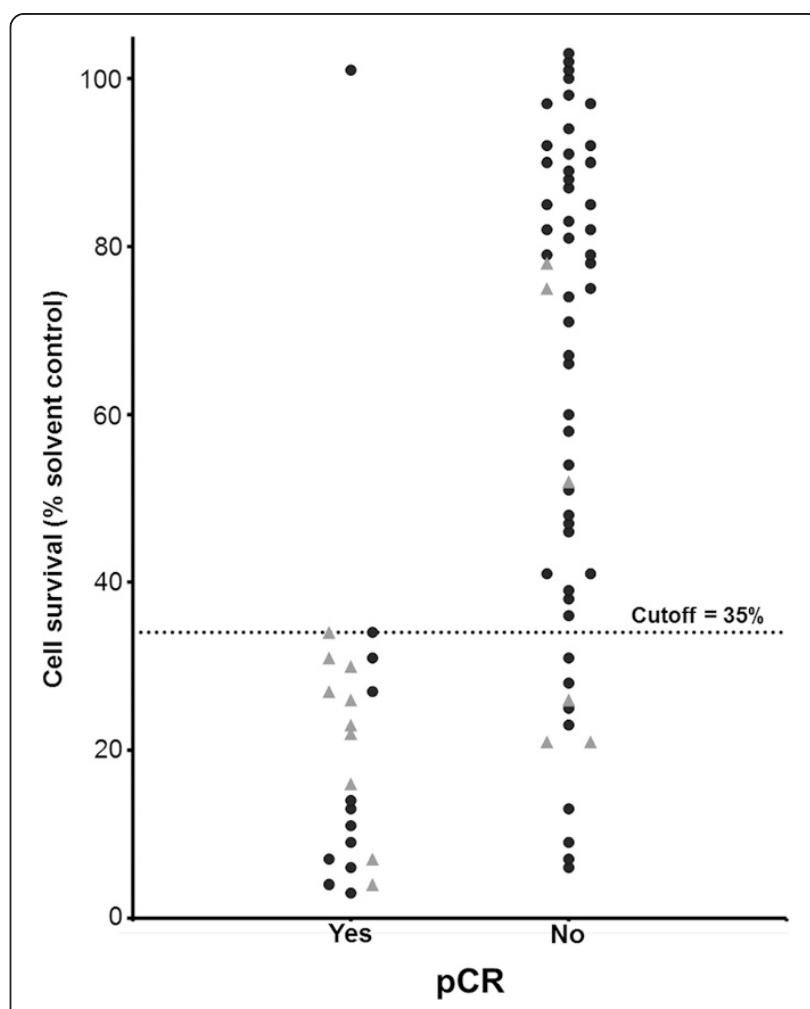

Fig. 2 Dot histogram showing the mean cell survival of each tumor sample following cytostatic treatment in the breast cancer spheroid model. The results are grouped according to $\mathrm{PCR}$. Each dot represents one patient; triangles represent patients treated with trastuzumab-based therapy. Proposed cutoff to predict pCR is shown at $35 \%$ cell survival 
Table 3 Correlation of cell survival with Clinical and Pathological Factors

\begin{tabular}{|c|c|c|c|c|c|c|c|}
\hline \multirow[b]{3}{*}{ Characteristics } & & & \multicolumn{4}{|c|}{ Cutoff } & \multirow[b]{3}{*}{$P$} \\
\hline & \multicolumn{2}{|c|}{ Mean } & \multicolumn{2}{|c|}{$<35 \%$} & \multicolumn{2}{|c|}{$\geq 35 \%$} & \\
\hline & $\%$ & $P$ & $\bar{n}$ & $\%$ & $\bar{n}$ & $\%$ & \\
\hline All Patients & & & 32 & 41.0 & 46 & 59.0 & \\
\hline Age at diagnosis, years & & .877 & & & & & 1.000 \\
\hline$\leq 50$ & 51.44 & & 18 & 41.9 & 25 & 58.1 & \\
\hline$>50$ & 52.61 & & 14 & 40.0 & 21 & 60.0 & \\
\hline Mean & - & & 51 & & 51 & & .817 \\
\hline Range & - & & $21-7$ & & $25-7$ & & \\
\hline Tumor stage & & .181 & & & & & .603 \\
\hline $\mathrm{CT} 1 / \mathrm{T} 2$ & 49.40 & & 24 & 42.9 & 32 & 57.1 & \\
\hline $\mathrm{CT} 3 / 4$ & 60.66 & & 7 & 33.3 & 14 & 66.7 & \\
\hline Nodal status & & .813 & & & & & 1.000 \\
\hline $\mathrm{cN}+$ & 50.41 & & 17 & 40.5 & 25 & 59.5 & \\
\hline $\mathrm{cN}-$ & 52.20 & & 15 & 42.9 & 20 & 57.1 & \\
\hline Grading & & .339 & & & & & .486 \\
\hline $\mathrm{G} 1 / 2$ & 55.34 & & 15 & 36.6 & 26 & 63.4 & \\
\hline G3 & 48.09 & & 16 & 45.7 & 19 & 54.3 & \\
\hline Not documented & & & - & & - & & \\
\hline Histologic type & & .569 & & & & & .439 \\
\hline Ductal invasive/other & 51.25 & & 30 & 42.9 & 40 & 57.1 & \\
\hline Lobular invasive & 58.29 & & 2 & 25.0 & 6 & 75.0 & \\
\hline HR status & & .038 & & & & & .042 \\
\hline $\mathrm{ER}+/ \mathrm{PR}+$ & 62.49 & & 10 & 25.6 & 29 & 74.4 & \\
\hline ER+/PR-/Unknown & 36.10 & & 7 & 70.0 & 3 & 30.0 & \\
\hline ER-/unknown/PR+ & 47.82 & & 1 & 50.0 & 1 & 50.0 & \\
\hline ER-/PR- & 43.03 & & 12 & 50.0 & 12 & 50.0 & \\
\hline HER2 status & & .000 & & & & & $<.0001$ \\
\hline Negative & 58.34 & & 17 & 28.8 & 42 & 71.2 & \\
\hline Positive & 30.70 & & 13 & 81.3 & 3 & 18.8 & \\
\hline
\end{tabular}

$\mathrm{PR}$, progesterone receptor; $\mathrm{ER}$, estrogen receptor; HER2, human epidermal growth factor receptor; $\mathrm{pCR}$, pathologic complete response

based therapy experienced higher pCR rates (Fig. 3c). Patients who did not receive the planned therapy had a lower rate of $\mathrm{pCR}$ (odds ratio $=-2.94, P=.0126$ ) The AUC from the model considering assay results and treatment adherence exhibited an AUC of 0.91 (Fig. 3d).

In addition to the pCR, correlation of cell survival with the ypT-stage revealed that the breast cancer spheroid model was significantly related to the gradual response as seen in the surgical specimen after chemotherapy (Fig. 4; Spearman's rho $=.311, p=.021$ ).

A cutoff associated with $\mathrm{pCR}$ in the breast cancer spheroid model was detected at $35 \%$ cell survival. This cutoff demonstrated a sensitivity of $95.5 \%$ (21 out of 22 ) and a specificity of $80.4 \%$ (45 out of 56 ).
Correlation of clinical and pathological data with the cutoff showed similar results in comparison to the reported correlations with mean cell survival (Table 3).

Odds ratios calculated with clinical and laboratory subgroups confirmed that HER2 status, treatment adherence, and cutoff in the breast cancer spheroid model significantly impact pCR (Table 4).

\section{Discussion}

The results of the SpheroNEO Study indicate that the in vitro breast cancer spheroid model correctly identified outcome for each treatment combination on an individual patient basis. Tumor spheroids reflect the cancer biology of the individual tumor more accurately compared to traditional 2-D cell assays. A tumor spheroid model, such as the one tested here, simulates the heterogeneity of cell types, cell-cell interactions, and the microenvironment of the patient tumor much more closely as compared to cell monolayer assays [44-46], in addition, through the 3-D structure a penetration barrier is formed allowing different concentrations of cytostatic agent to reach each tumor cell [47].

Cell-based chemosensitivity/chemoresistence assays have been tested since the early 1970s, and the results seemed initially promising $[28,32,48,49]$. However, implementation of these assays into the clinical routine was not successful $[32,50,51]$. With an abundance of available drugs and targeted therapies today, the number of available treatment options for each patient is increasing, and there is a necessity for a preclinical model to stratify each patient to the optimal treatment option [52].

The aim of the present study was to introduce a preclinical in vitro assay to tailor the best possible anti-tumor treatment for breast cancer patients in the neoadjuvant setting. There are some limitations regarding laboratory methodology. The main difficulty was the minimal number of cells required for a reliable assay outcome. The methodology had previously been established using surgical specimen from colorectal carcinoma patients, which consistently yielded a greater number of isolated cells. Adapting this method to the much smaller breast cancer biopsies proved challenging, especially since the anatomy of the breast is much more diverse in its tissue components. This resulted in a wide range of total isolated cell number per sample weight.

A second factor that influenced the drop-out rate of patients was due to the timeline of the neoadjuvant treatment. At the time of biopsy for the initial histological confirmation no final decision regarding the chemotherapy regimen had been made for the respective breast cancer patient. This explains the considerable number of patients beginning a different neoadjuvant treatment as initially planned, receiving primary surgery with or without adjuvant treatment instead. The final 


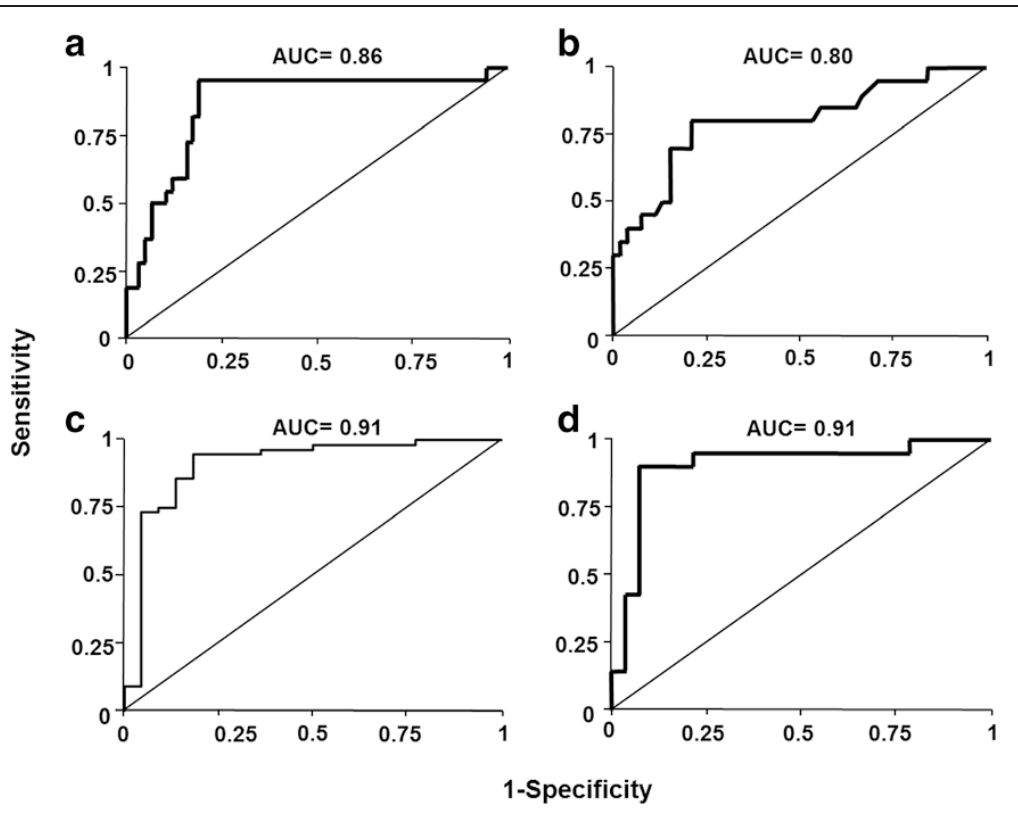

Fig. 3 Receiver operator curves (ROC) displaying the sensitivity and specificity of (a) the breast cancer spheroid, (b) a multifactor model with baseline predictive factors (ER, PR, HER2, and age) impacting pCR, (c) model combining the breast cancer spheroid model, HER2-Status and type of therapy, and (d) breast cancer spheroid model combined with the factor of treatment adherence defined as treatment discontinuation, dose-reduction or change of treatment. The resulting area under the curve (AUC) is displayed at the top of each curve

treatment decision is made in collaboration between patient and physician, taking many clinical and nononcologic factors into account. Treatment non-adherence was most frequent in older women, as well as in patients with one or multiple comorbidities.

In vitro treatment efficacy results comparing standard treatment combinations for all patients in this study recapitulate treatment options as outlined for various breast cancer subgroups in current guidelines. The high standard deviations of these results reflect the heterogeneity of the patient subgroups. A total of six patients were identified where more than one treatment combination could be tested. The results of the breast cancer spheroid model correctly identified the efficacy of the clinical treatment combination by demonstrating a significantly lower cell survival compared to other treatment combinations tested. Interestingly at least two or more treatment combinations proved equally effective in vitro, implying

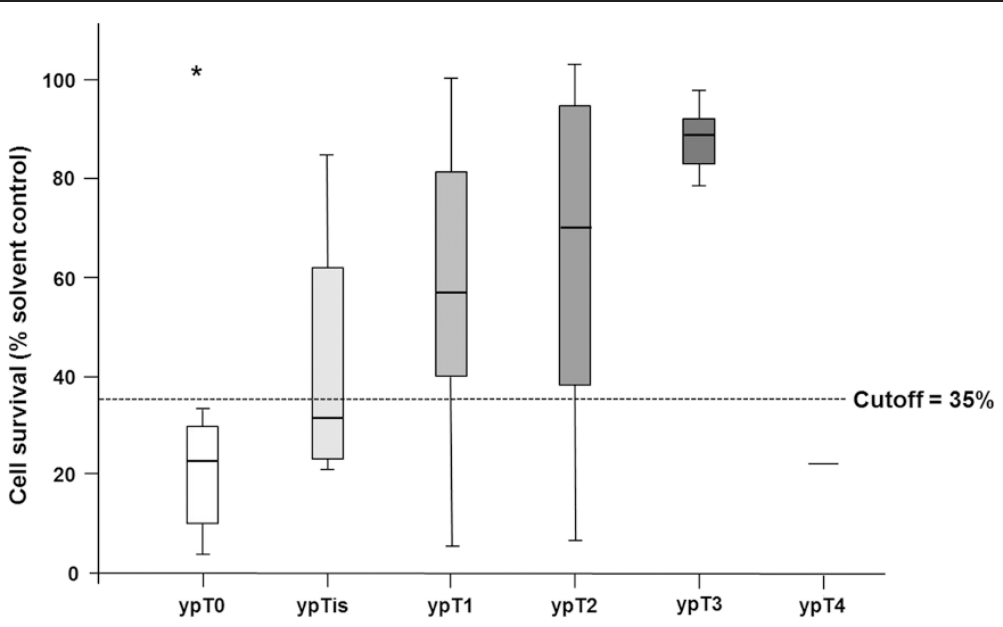

Fig. 4 Box plot diagram showing mean cell survival in the breast cancer spheroid model after cytostatic treatment in comparison to the remaining tumor as determined by the pathological assessment of the surgical specimen after chemotherapy. Proposed cutoff of $35 \%$ is represented by the dotted line. ypTO/ypNo $=24.4 \%(n=19)$, ypTis $=5.1 \%(n=4$; ypNO 2 out of 3), ypT1 = 33.3\% ( $n=26)$, ypT2= $20.5 \%(n=16)$, ypT3=10.3 \% $(n=8)$, ypT4 $=1.3 \%(n=1)$, not documented $(n=4)$, * represents an outlier 
Table 4 Odds ratios for the clinical and laboratory variables impacting $\mathrm{pCR}$ in a multivariable model

\begin{tabular}{lcll}
\hline Characteristics & Odds ratio & $95 \% \mathrm{Cl}$ & $P$ \\
\hline Cell survival cutoff $(>35 \% / \leq 35 \%)$ & 0.011 & $0.001-0.096$ & .0001 \\
Treatment adherence (no/yes) & 0.115 & $0.014-0.925$ & .0421 \\
HR status (pos/neg) & 0.513 & $0.180-1.465$ & .2123 \\
HER2 status (neg/pos) & 0.167 & $0.050-0.560$ & .0037 \\
Age $(>50 / \leq 50$ years) & 0.501 & $0.177-1.417$ & .1505 \\
\hline
\end{tabular}

that a decision between approved anthracyclines or taxanes could be made according to each patient's comorbidity or tolerability.

Furthermore, the breast cancer spheroid model showed a predictive response to trastuzumab-based therapy prior to histopathological confirmation of the HER2 status. In the tumor samples tested in vitro with trastuzumab-based therapy, a selective effect was seen. If the in vitro treatment with trastuzumab showed no additional benefit as compared to chemotherapy alone, the patients were histologically confirmed HER2 negative $(n=5)$.

\section{Conclusion}

These preliminary analyses indicate that the breast cancer spheroid model is not only predictive but might also be selective in discerning ineffective from effective treatment options.

In order to validate the results from this explorative study an interventional randomized controlled confirmatory study is planned, follow-up data will also be analyzed. The cutoff as seen in this cohort of patients will be analyzed in regard to its validity and reliability in a larger cohort of patients.

\section{Additional file}

Additional file 1: List of participating study sites and applicable ethics committees.

\section{Abbreviations}

AUC: Area under the curve; Cl: Confidence interval; cps: Counts per second; HER2: Human epidermal growth factor 2; mTOR: Mammalian target of rapamycin; pCR: Pathologic complete response; ppc: Peak plasma concentration; ROC: Receiver operator curve; TNR: True negative rate; TPR: True positive rate.

\section{Competing interests}

Employment or Leadership Position: Kathrin Halfter, Ilona Funke, Barbara Mayer, SpheroTec GmbH, Gunter von Minckwitz, German Breast Group Research $\mathrm{GmbH}$

Consultant or Advisory Role: Gunter von Minckwitz, SpheroTec GmbH, Astra Zeneca, Abbvie, Celgene, Pfizer, Roche, Illona Funke, SpheroTec GmbH, Nina Ditsch, Astra Zeneca, Hans-Christian Kolberg, Novartis, Holger Fischer, Roche Pharma

Stock Ownership: Ilona Funke, Barbara Mayer, SpheroTec GmbH, Gunter von Minckwitz, German Breast Group Research GmbH

Honoraria: Nina Ditsch, Myriad Genetics, Holger Fischer, Roche Pharma,

Gunter von Minckwitz, Amgen, Astra Zeneca, Roche
Funding: Ilona Funke, Alexander Crispin, SpheroTec GmbH, Hans-Christian Kolberg, Novartis and Amgen, Gunter von Minckwitz, Pfizer, GSK, Sanofi-Aventis, Amgen, Roche, Novartis, Celgene, Teva, Boehringer-Ingelheim Expert Testimony: None

Patents, Royalties, and Licenses: Ilona Funke, Barbara Mayer, SpheroTec $\mathrm{GmbH}$

Travel Expenses: Hans-Christian Kolberg, Novartis, GSK, Astra Zeneca and Teva, Holger Fischer, Roche Pharma and Teva, Ilona Funke,Barbara Mayer, SpheroTec $\mathrm{GmbH}$

No other conflicts of interest exist. All remaining authors have declared no conflicts of interest.

\section{Authors' contributions}

The study was designed and a synopsis was drafted by BM, IF and ND. Data acquisition and analysis of the laboratory and clinical data was done by $\mathrm{KH}$ and $A C$ at the University Clinic in Munich, Germany. Data interpretation was performed by ND, AC, and GVM. ND, HCK, HF, TH, FEvK and IB contributed significant numbers of patients for inclusion in the study. The first draft of the study report was written by $\mathrm{KH}$ and reviewed and approved by all co-authors before submission. Full access to the raw data was granted to BM and $A C$ at all times. All authors read and approved the final manuscript.

\section{Acknowledgment}

We would like to thank the patients who took part in the study, as well as the participating study teams for their outstanding cooperation. We would also like to thank Karin von Dehn-Rotfelser and Oliver Hoffman for their technical assistance, as well as Jana Peplinski and Silvia Paulick for support in data management.

\section{Role of the funding source}

The study was co-funded by academia and industry. The SpheroTec GmbH performed the laboratory testing for all samples and provided the raw laboratory data to the statistician (AC). The academic co-sponsor was not involved in any phase of the completion of the final paper being submitted.

\section{Author details}

${ }^{1}$ Department of General, Visceral, Transplantation, Vascular and Thoraic Surgery, Hospital of the University of Munich, Munich, Germany.

${ }^{2}$ Department of Obstetrics and Gynecology, Ludwig-Maximilians-University of Munich, Munich, Germany. ${ }^{3}$ Marienhospital Bottrop, Bottrop, Germany.

${ }^{4}$ Evangelische Kliniken Gelsenkirchen, Gelsenkirchen, Germany. ${ }^{5}$ Klinikum St. Marien Amberg, Amberg, Germany. ${ }^{6}$ Department of Obstetrics and Gynecology, Klinikum Dritter Orden, Munich, Germany. ${ }^{7}$ Klinikum Landshut, Landshut, Germany. ${ }^{8} \mathrm{GBG}$ Forschungs $\mathrm{GmbH}$, Neu-Isenburg and University Women's Hospital Frankfurt, Frankfurt, Germany. ${ }^{9}$ SpheroTec GmbH, Martinsried, Germany. ${ }^{10}$ IBE LMU, Department of Obstetrics and Gynecology, Technical University of Munich, Klinikum Starnberg, Leopoldina Krankenhaus der Stadt Schweinfurt, Markus Krankenhaus Frankfurt, Klinikum Nürnberg, Städtisches Klinkum Karlsruhe, Klinikum Harlaching, Munich, Germany.

Received: 9 September 2014 Accepted: 16 June 2015

Published online: 15 July 2015

\section{References}

1. von Minckwitz G, Untch M, Blohmer JU, Costa SD, Eidtmann H, Fasching PA, et al. Definition and impact of pathologic complete response on prognosis after neoadjuvant chemotherapy in various intrinsic breast cancer subtypes. J Clin Oncol. 2012;30(15):1796-804.

2. Esserman LJ, Berry DA, DeMichele A, Carey L, Davis SE, Buxton M, et al. Pathologic complete response predicts recurrence-free survival more effectively by cancer subset: results from the I-SPY 1 TRIAL-CALGB 150007/150012, ACRIN 6657. J Clin Oncol. 2012;30(26):3242-9.

3. Rastogi P, Anderson SJ, Bear HD, Geyer CE, Kahlenberg MS, Robidoux A, et al. Preoperative chemotherapy: updates of National Surgical Adjuvant Breast and Bowel Project Protocols B-18 and B-27. J Clin Oncol. 2008;26(5):778-85.

4. Kuerer HM, Newman LA, Smith TL, Ames FC, Hunt KK, Dhingra K, et al. Clinical course of breast cancer patients with complete pathologic primary tumor and axillary lymph node response to doxorubicin-based neoadjuvant chemotherapy. J Clin Oncol. 1999;17(2):460-9. 
5. Prowell TM, Pazdur R. Pathological complete response and accelerated drug approval in early breast cancer. N Engl J Med. 2012;366(26):2438-41.

6. Cortazar P, Zhang L, Untch M, Mehta K, Costantino JP, Wolmark N, et al. Pathological complete response and long-term clinical benefit in breast cancer: the CTNeoBC pooled analysis. Lancet. 2014;384(9938):164-72.

7. Montagna E, Bagnardi V, Rotmensz N, Viale G, Pruneri G, Veronesi P, et al. Pathological complete response after preoperative systemic therapy and outcome: relevance of clinical and biologic baseline features. Breast Cancer Res Treat. 2010;124(3):689-99.

8. von Minckwitz G, Kummel S, Vogel P, Hanusch C, Eidtmann H, Hilfrich J, et al. Intensified neoadjuvant chemotherapy in early-responding breast cancer: phase III randomized GeparTrio study. J Natl Cancer Inst. 2008;100(8):552-62.

9. Earl HM, Vallier AL, Hiller L, Fenwick N, Young J, Iddawela M, et al. Effects of the addition of gemcitabine, and paclitaxel-first sequencing, in neoadjuvant sequential epirubicin, cyclophosphamide, and paclitaxel for women with high-risk early breast cancer (Neo-tAnGo): an open-label, 2×2 factorial randomised phase 3 trial. Lancet Oncol. 2014;15(2):201-12.

10. Buzdar AU, Suman VJ, Meric-Bernstam F, Leitch AM, Ellis MJ, Boughey JC, et al. Fluorouracil, epirubicin, and cyclophosphamide (FEC-75) followed by paclitaxel plus trastuzumab versus paclitaxel plus trastuzumab followed by FEC-75 plus trastuzumab as neoadjuvant treatment for patients with HER2-positive breast cancer (Z1041): a randomised, controlled, phase 3 trial. Lancet Oncol. 2013;14(13):1317-25.

11. Connolly RM, Stearns V. Current approaches for neoadjuvant chemotherapy in breast cancer. Eur J Pharmacol. 2013;717(1-3):58-66.

12. Gerber B, Loibl S, Eidtmann H, Rezai M, Fasching PA, Tesch $H$, et al. Neoadjuvant bevacizumab and anthracycline-taxane-based chemotherapy in 678 triple-negative primary breast cancers; results from the geparquinto study (GBG 44). Ann Oncol. 2013;24(12):2978-84

13. Alba E, Albanell J, de la Haba J, Barnadas A, Calvo L, Sanchez-Rovira P, et al. Trastuzumab or lapatinib with standard chemotherapy for HER2-positive breast cancer: results from the GEICAM/2006-14 trial. Br J Cancer. 2014;1 10(5):1139-47.

14. von Minckwitz G, Rezai M, Loibl S, Fasching PA, Huober J, Tesch H, et al. Capecitabine in addition to anthracycline- and taxane-based neoadjuvant treatment in patients with primary breast cancer: phase III GeparQuattro study. Ann Oncol. 2010;28(12):2015-23.

15. Huober J, Fasching PA, Hanusch C, Rezai M, Eidtmann H, Kittel K, et al. Neoadjuvant chemotherapy with paclitaxel and everolimus in breast cancer patients with non-responsive tumours to epirubicin/cyclophosphamide (EC) +/- bevacizumab - results of the randomised GeparQuinto study (GBG 44). Eur J Cancer. 2013;49(10):2284-93.

16. Untch M, Loibl S, Bischoff J, Eidtmann H, Kaufmann M, Blohmer JU, et al. Lapatinib versus trastuzumab in combination with neoadjuvant anthracycline-taxane-based chemotherapy (GeparQuinto, GBG 44): a randomised phase 3 trial. Lancet Oncol. 2012;13(2):135-44.

17. von Minckwitz G, Schneeweiss A, Loibl S, Salat C, Denkert C, Rezai M, et al. Neoadjuvant carboplatin in patients with triple-negative and HER2-positive early breast cancer (GeparSixto; GBG 66): a randomised phase 2 trial. Lancet Oncol. 2014;15(7):747-56

18. Buzdar AU, Ibrahim NK, Francis D, Booser DJ, Thomas ES, Theriault RL, et al Significantly higher pathologic complete remission rate after neoadjuvant therapy with trastuzumab, paclitaxel, and epirubicin chemotherapy: results of a randomized trial in human epidermal growth factor receptor 2-positive operable breast cancer. J Clin Oncol. 2005;23(16):3676-85.

19. Dent S, Oyan B, Honig A, Mano M, Howell S. HER2-targeted therapy in breast cancer: a systematic review of neoadjuvant trials. Cancer Treat Rev. 2013;39(6):622-31.

20. Gianni L, Pienkowski T, Im YH, Roman L, Tseng LM, Liu MC, et al. Efficacy and safety of neoadjuvant pertuzumab and trastuzumab in women with locally advanced, inflammatory, or early HER2-positive breast cancer (NeoSphere): a randomised multicentre, open-label, phase 2 trial. Lancet Oncol. 2012;13(1):25-32.

21. Pohlmann PR, Mayer IA, Mernaugh R. Resistance to Trastuzumab in Breast Cancer. Clin Cancer Res. 2009;15(24):7479-91.

22. Wilson S, Chia S. New agents in locally advanced breast cancer. Curr Opin Support Palliat Care. 2014;8(1):64-9.

23. Slamon DJ, Leyland-Jones B, Shak S, Fuchs H, Paton V, Bajamonde A, et al. Use of chemotherapy plus a monoclonal antibody against HER2 for metastatic breast cancer that overexpresses HER2. N Engl J Med. 2001;344(11):783-92.

24. Kittaneh M, Montero AJ, Gluck S. Molecular Profiling for Breast Cancer: A Comprehensive Review. Biomark Cancer. 2013;5:61-70.
25. Azim Jr HA, Michiels S, Bedard PL, Singhal SK, Criscitiello C, Ignatiadis M, et al. Elucidating prognosis and biology of breast cancer arising in young women using gene expression profiling. Clin Cancer Res. 2012;18(5):1341-51.

26. Paik S, Tang G, Shak S, Kim C, Baker J, Kim W, et al. Gene expression and benefit of chemotherapy in women with node-negative, estrogen receptor-positive breast cancer. J Clin Oncol. 2006:24(23):3726-34.

27. Harbeck N, Sotlar K, Wuerstlein R, Doisneau-Sixou S. Molecular and protein markers for clinical decision making in breast cancer: Today and tomorrow. Cancer Treat Rev. 2014;40(3):434-44.

28. Mi Z, Holmes FA, Hellerstedt B, Pippen J, Collea R, Backner A, et al. Feasibility assessment of a chemoresponse assay to predict pathologic response in neoadjuvant chemotherapy for breast cancer patients. Anticancer Res. 2008;28(3B):1733-40.

29. Loizzi V, Chan JK, Osann K, Cappuccini F, DiSaia PJ, Berman ML. Survival outcomes in patients with recurrent ovarian cancer who were treated with chemoresistance assay-guided chemotherapy. Am J Obstet Gynecol. 2003;189(5):1301-7.

30. Kern DH, Weisenthal LM. Highly specific prediction of antineoplastic drug resistance with an in vitro assay using suprapharmacologic drug exposures. J Natl Cancer Inst. 1990;82(7):582-8.

31. Sharma S, Neale MH, Di Nicolantonio F, Knight LA, Whitehouse PA, Mercer SJ, et al. Outcome of ATP-based tumor chemosensitivity assay directed chemotherapy in heavily pre-treated recurrent ovarian carcinoma. BMC Cancer. 2003;3:19.

32. Burstein HJ, Mangu PB, Somerfield MR, Schrag D, Samson D, Holt L, et al. American Society of Clinical Oncology clinical practice guideline update on the use of chemotherapy sensitivity and resistance assays. J Clin Oncol. 2011;29(24):3328-30.

33. Vidi PA, Bissell MJ, Lelievre SA. Three-dimensional culture of human breast epithelial cells: the how and the why. Methods Mol Biol. 2013;945:193-219.

34. Nagelkerke A, Bussink J, Sweep FC, Span PN. Generation of multicellular tumor spheroids of breast cancer cells: how to go three-dimensional. Anal Biochem. 2013:437(1):17-9.

35. Weiswald LB, Richon S, Massonnet G, Guinebretiere JM, Vacher S, Laurendeau I, et al. A short-term colorectal cancer sphere culture as a relevant tool for human cancer biology investigation. Br J Cancer. 2013;108(8):1720-31.

36. Napolitano AP, Dean DM, Man AJ, Youssef J, Ho DN, Rago AP, et al. Scaffold-free three-dimensional cell culture utilizing micromolded nonadhesive hydrogels. BioTechniques. 2007;43(4):494. 496-500.

37. Kondo J, Endo H, Okuyama H, Ishikawa O, lishi H, Tsujii M, et al. Retaining cell-cell contact enables preparation and culture of spheroids composed of pure primary cancer cells from colorectal cancer. Proc Natl Acad Sci U S A. 2011;108(15):6235-40.

38. Tung YC, Hsiao AY, Allen SG, Torisawa YS, Ho M, Takayama S. High-throughput $3 \mathrm{D}$ spheroid culture and drug testing using a 384 hanging drop array. Analyst. 2011;136(3):473-8

39. Yuhas JM, Li AP, Martinez AO, Ladman AJ. A simplified method for production and growth of multicellular tumor spheroids. Cancer Res. 1977;37(10):3639-43.

40. Mayer B, Klement G, Kaneko M, Man S, Jothy S, Rak J, et al. Multicellular gastric cancer spheroids recapitulate growth pattern and differentiation phenotype of human gastric carcinomas. Gastroenterology. 2001;121(4):839-52.

41. Angelucci D, Tinari N, Grassadonia A, Cianchetti E, Ausili-Cefaro G, lezzi L, et al. Long-term outcome of neoadjuvant systemic therapy for locally advanced breast cancer in routine clinical practice. J Cancer Res Clin Oncol. 2013;139(2):269-80

42. Untch M, Fasching PA, Konecny GE, von Koch F, Conrad U, Fett W, et al. PREPARE trial: a randomized phase III trial comparing preoperative, dose-dense, dose-intensified chemotherapy with epirubicin, paclitaxel and CMF versus a standard-dosed epirubicin/cyclophosphamide followed by paclitaxel +/darbepoetin alfa in primary breast cancer-results at the time of surgery. Ann Oncol. 2011;22(9):1988-98.

43. Hoffmann Ol, Imberger $C$, Magosch $\mathrm{S}$, Joka M, Jauch KW, Mayer B. Impact of the spheroid model complexity on drug response. J Biotechnol. 2015;205:14-23.

44. Kim S, Alexander CM: Tumorsphere assay provides more accurate prediction of in vivo responses to chemotherapeutics. Biotechnology letters 2013:36:481-488.

45. Mehta G, Hsiao AY, Ingram M, Luker GD, Takayama S. Opportunities and challenges for use of tumor spheroids as models to test drug delivery and efficacy. J Control Release. 2012;164(2):192-204. 
46. Herrmann D, Conway JR, Vennin C, Magenau A, Hughes WG, Morton JP, et al. Three-dimensional cancer models mimic cell-matrix interactions in the tumour microenvironment. Carcinogenesis. 2014;35(8):1671-9.

47. Minchinton Al, Tannock IF. Drug penetration in solid tumours. Nat Rev Cancer. 2006;6(8):583-92.

48. Cortazar P, Johnson BE. Review of the efficacy of individualized chemotherapy selected by in vitro drug sensitivity testing for patients with cancer. J Clin Oncol. 1999;17(5):1625-31.

49. Bertelsen CA, Sondak VK, Mann BD, Korn EL, Kern DH. Chemosensitivity testing of human solid tumors. A review of 1582 assays with 258 clinical correlations. Cancer. 1984;53(6):1240-5.

50. Rutherford T, Orr Jr J, Grendys Jr E, Edwards R, Krivak TC, Holloway R, et al. A prospective study evaluating the clinical relevance of a chemoresponse assay for treatment of patients with persistent or recurrent ovarian cancer. Gynecol Oncol. 2013;131(2):362-7.

51. Nagourney RA, Blitzer JB, Shuman RL, Asciuto TJ, Deo EA, Paulsen M, et al. Functional profiling to select chemotherapy in untreated, advanced or metastatic non-small cell lung cancer. Anticancer Res. 2012;32(10):4453-60.

52. Eccles SA, Aboagye EO, Ali S, Anderson AS, Armes J, Berditchevski F, et al. Critical research gaps and translational priorities for the successful prevention and treatment of breast cancer. Breast Cancer Res. 2013;15(5):R92.

53. Go RS, Adjei AA. Review of the comparative pharmacology and clinical activity of cisplatin and carboplatin. J Clin Oncol. 1999;17(1):409-22.

54. Egorin MJ, Forrest A, Belani CP, Ratain MJ, Abrams JS, Van Echo DA. A limited sampling strategy for cyclophosphamide pharmacokinetics. Cancer Res. 1989;49(11):3129-33.

55. Baker SD, Zhao M, Lee CK, Verweij J, Zabelina Y, Brahmer JR, et al. Comparative pharmacokinetics of weekly and every-three-weeks docetaxel. Clin Cancer Res. 2004;10(6):1976-83.

56. Bruno R, Hille D, Riva A, Vivier N, ten Bokkel Huinnink WW, van Oosterom AT, et al. Population pharmacokinetics/pharmacodynamics of docetaxel in phase II studies in patients with cancer. J Clin Oncol. 1998;16(1):187-96.

57. Brana I, Ocana A, Chen EX, Razak AR, Haines C, Lee C, et al. A phase I trial of pantoprazole in combination with doxorubicin in patients with advanced solid tumors: evaluation of pharmacokinetics of both drugs and tissue penetration of doxorubicin. Investig New Drugs. 2014;32(6):1269-77.

58. Fujimoto S. Promising antitumor activity of a novel quinoline derivative, TAS-103, against fresh clinical specimens of eight types of tumors measured by flow cytometric DNA analysis. Biol Pharm Bull. 2007;30(10):1923-9.

59. Gianni L, Kearns CM, Giani A, Capri G, Vigano L, Lacatelli A, et al. Nonlinear pharmacokinetics and metabolism of paclitaxel and its pharmacokinetic/ pharmacodynamic relationships in humans. J Clin Oncol. 1995;13(1):180-90.

60. Leyland-Jones B. Dose scheduling-Herceptin. Oncology. 2001;61 Suppl 2:31-6.

\section{Submit your next manuscript to BioMed Central and take full advantage of:}

- Convenient online submission

- Thorough peer review

- No space constraints or color figure charges

- Immediate publication on acceptance

- Inclusion in PubMed, CAS, Scopus and Google Scholar

- Research which is freely available for redistribution

Submit your manuscript at www.biomedcentral.com/submit 